\title{
Hyphenation of a EC / OC thermal-optical carbon analyzer to photo ionization time-of-flight mass spectrometry: a new off-line aerosol mass spectrometric approach for characterization of primary and secondary particulate matter
}

\section{J. Diab et al.}

Correspondence to: R. Zimmermann (ralf.zimmermann@uni-rostock.de) 


\section{Hyphenation of a EC / OC thermal-optical carbon analyzer to photo ionization time-of-flight mass spectrometry: a new off-line aerosol mass spectrometric approach for characterization of primary and secondary particulate matter}

\section{J. Diab et al.}

Correspondence to: R. Zimmermann (ralf.zimmermann@uni-rostock.de) 


\section{Supplemental material}

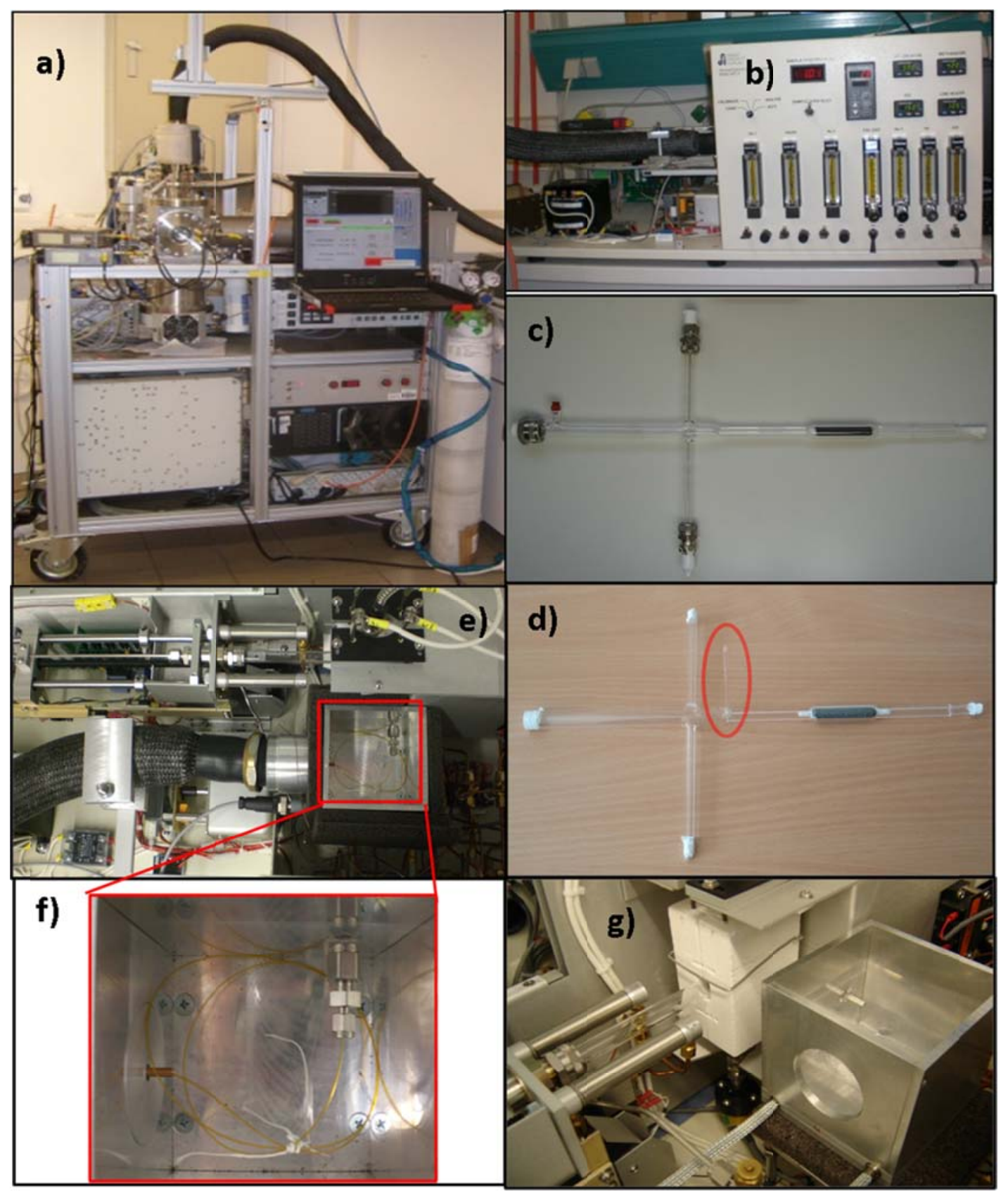

Figure S 1: pictures of the coupling and assembling (a- PI-TOF-MS, b - carbon analyzer, c - the original quartz tubing system, $d$ - the modified quartz tubing system, e - the whole connecting unit with aluminum box and heating hose, $\mathrm{f}$ - zoom into the aluminum box with quartz tube, Swagelok connector and transfer capillary, $\mathrm{g}$ - quartz tube reaching into the aluminum box with heating cartridges) 
Table S1: experiment conditions of the chamber experiments

\begin{tabular}{lcccccc}
\hline & $\begin{array}{c}\text { Precursor } \\
\text { concentration } \\
{[\mathrm{ppm}]}\end{array}$ & $\begin{array}{c}\text { Ozone } \\
\text { concentration } \\
{[\mathrm{ppm}]}\end{array}$ & $\begin{array}{c}\text { Temperature } \\
{\left[{ }^{\circ} \mathrm{C}\right]}\end{array}$ & $\begin{array}{c}\text { chamber } \\
\text { volume } \\
{\left[\mathrm{m}^{3}\right]}\end{array}$ & $\begin{array}{c}\text { Experiment } \\
\text { duration } \\
{[\mathrm{min}]}\end{array}$ & $\begin{array}{c}\text { PM on } \\
\text { filter } \\
{\left[\mu \mathrm{g} / \mathrm{cm}^{2}\right]}\end{array}$ \\
\hline \multicolumn{2}{l}{ Filter Samples Hong Kong } & & & & & \\
$\begin{array}{l}\alpha \text {-pinene } \\
\beta \text {-pinene }\end{array}$ & 500 & 0.069 & 23 & 18.26 & 135 & 51.8 \\
Isoprene & 200 & 0.157 & 23 & 18.26 & 135 & 15.2 \\
Filter samples Karlsruhe & 100 & 0.17 & 23 & 18.26 & 240 & 12.9 \\
$\alpha$-pinene I & 2.2 & 25 & 22 & 3.7 & 55 & n.a. \\
$\alpha$-pinene II & 2.215 & 6 & 24 & 3.7 & 30 & n.a.
\end{tabular}

Table S2: Carbon contents [ $\mu \mathrm{g} /$ filter] of all samples corrected with laser transmittance (with regular split)

\begin{tabular}{|c|c|c|c|c|c|c|}
\hline sample & $\mathrm{TC}[\mu \mathrm{g} /$ filter $]$ & OC $[\mu \mathrm{g} /$ filter $]$ & $\mathrm{EC}[\mu \mathrm{g} /$ filter $]$ & OC/EC & OC/TC [\%] & EC/TC [\%] \\
\hline diesel & 24.18 & 12.47 & 11.71 & 1.07 & 52 & 48 \\
\hline gasoline & 33.54 & 8.06 & 25.49 & 0.32 & 24 & 76 \\
\hline wood combustion I & 130.56 & 122.63 & 7.92 & 15.48 & 94 & 6 \\
\hline wood combustion II & 67.75 & 51.25 & 16.50 & 3.11 & 76 & 24 \\
\hline Ispra winter & 25.18 & 18.61 & 6.57 & 2.83 & 74 & 26 \\
\hline Ispra summer & 4.25 & 3.62 & 0.63 & 5.75 & 85 & 15 \\
\hline$\beta$-pinene HK & 3.27 & 3.27 & 0.00 & $\infty$ & 100 & 0 \\
\hline$\alpha$-pinene HK & 12.66 & 12.65 & 0.01 & 1264.50 & 100 & 0 \\
\hline$\alpha$-pinene KR & 28.01 & 27.98 & 0.03 & 932.67 & 100 & 0 \\
\hline isoprene HK & 0.56 & 0.55 & 0.01 & 55.00 & 98 & 2 \\
\hline
\end{tabular}



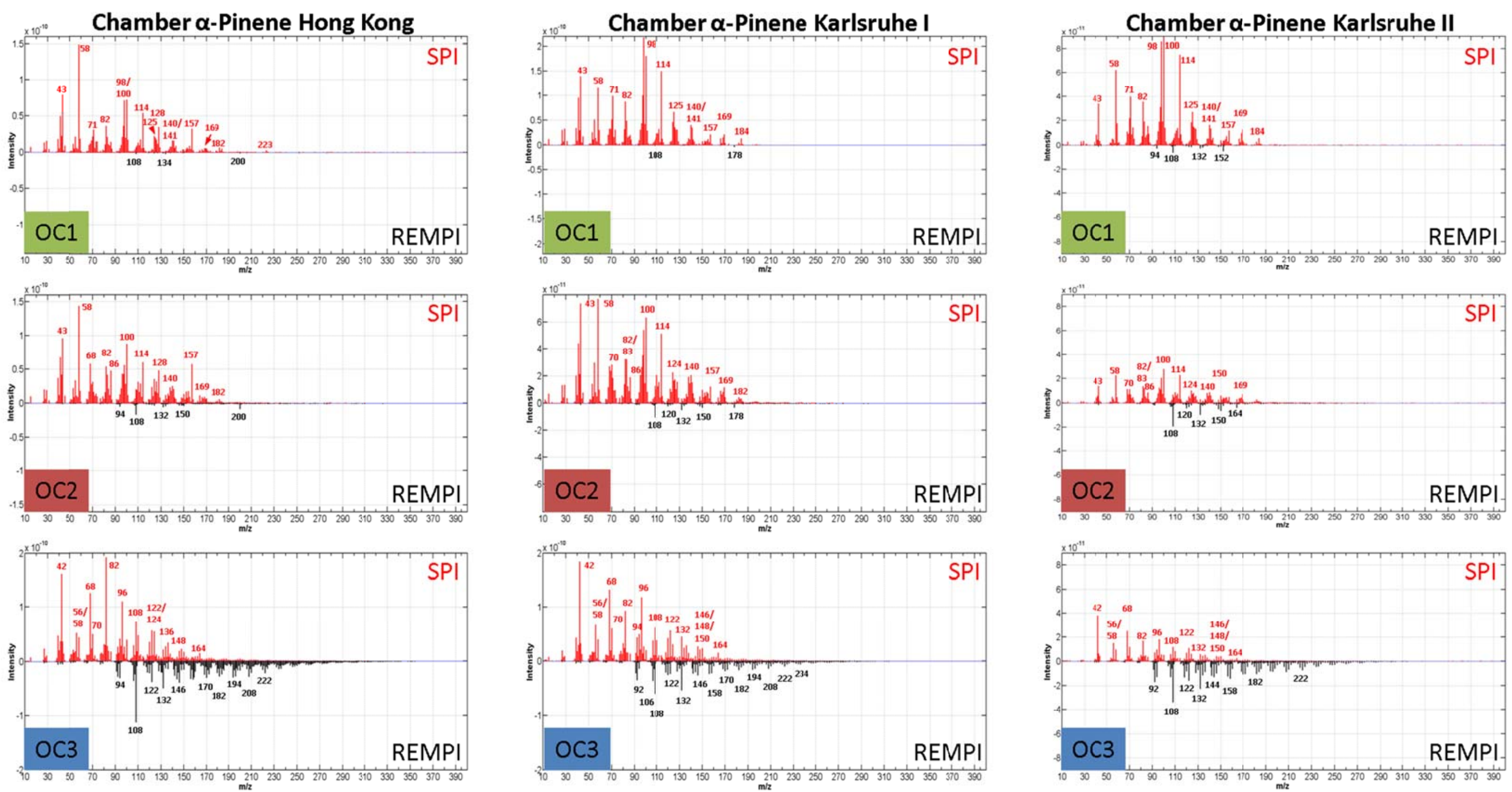

Figure S 2: Comparison of the mass spectra obtained by the measurement of the filter samples from the three different chamber experiments using $\alpha$ pinene as a precursor. 

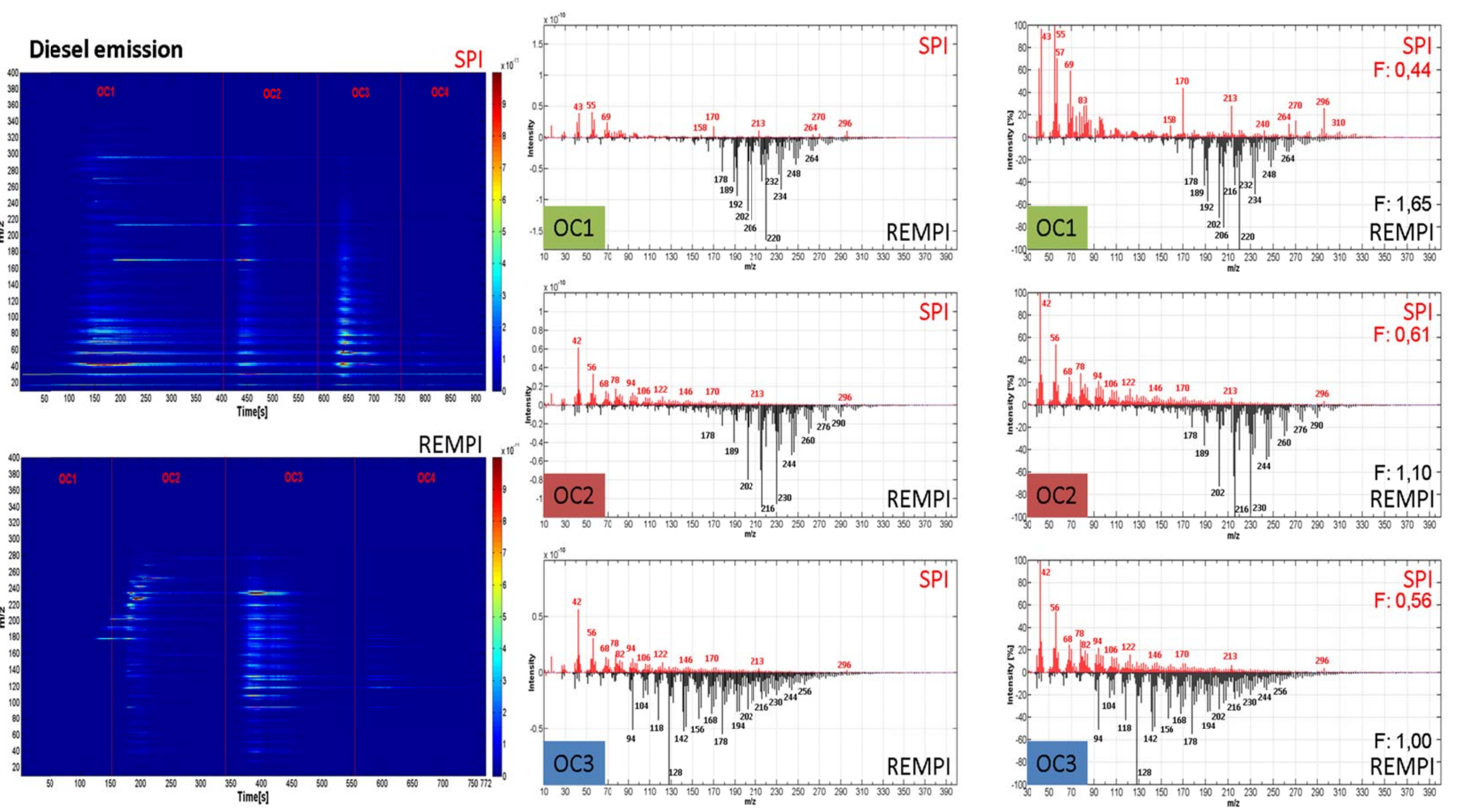

Figure S3: Two-dimensional mass spectrum of the course of signals ( $\mathrm{m} / \mathrm{z}$ ) over the four OC-steps (left) for SPI (top) and REMPI (bottom), mass spectra for the first three OC-steps (SPI-red, REMPI-black) (middle), mass spectra for the first three OC-steps normalized to $100 \%$ (right) of the diesel emission sample . Measured with the IMPROVE protocol. 

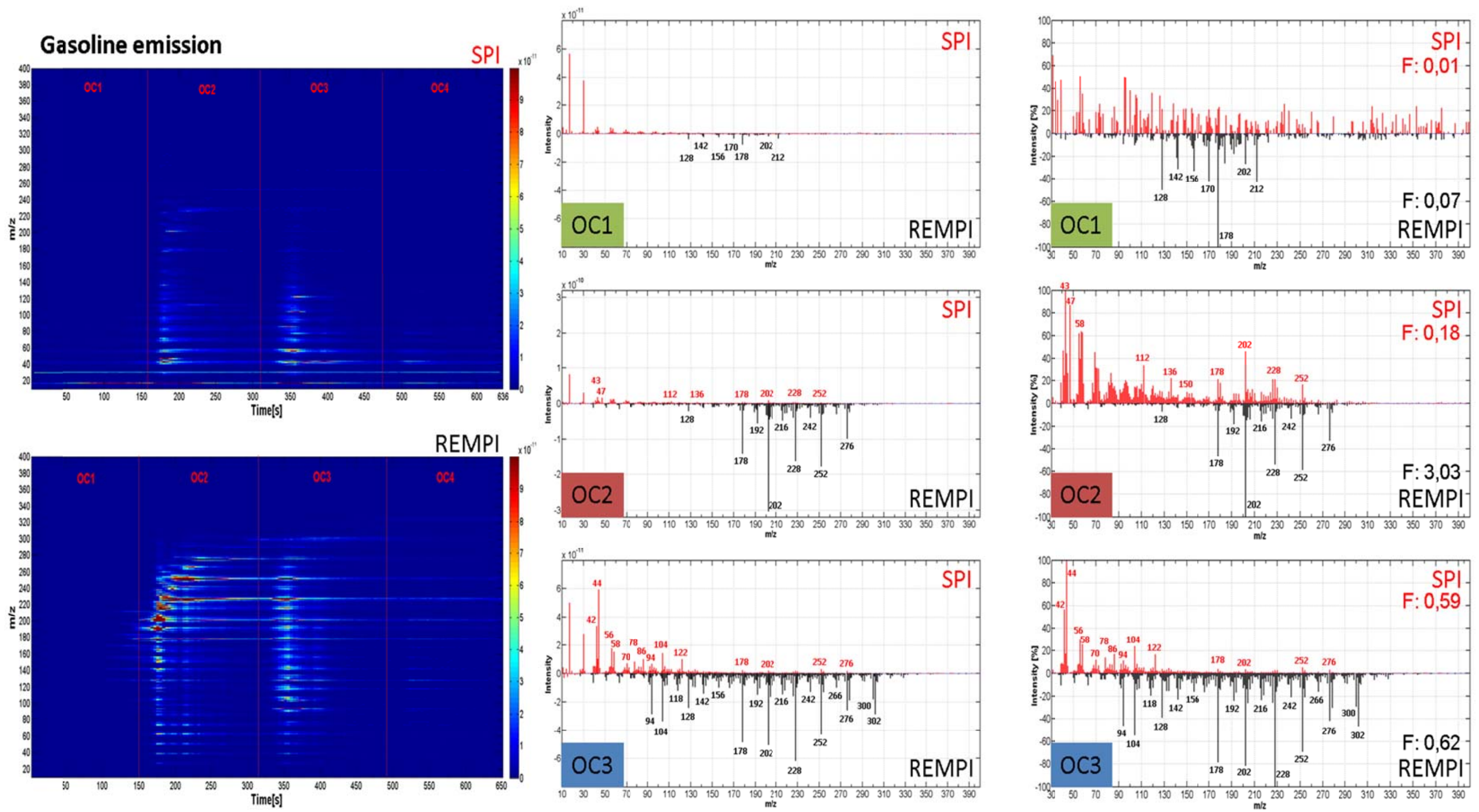

Figure S4: Two-dimensional mass spectrum of the course of signals ( $\mathrm{m} / \mathrm{z}$ ) over the four OC-steps (left) for SPI (top) and REMPI (bottom), mass spectra for the first three OC-steps (SPI-red, REMPI-black) (middle), mass spectra for the first three OC-steps normalized to $100 \%$ (right) of the gasoline emission sample. Measured with the IMPROVE protocol. 


\section{Wood combustion I}
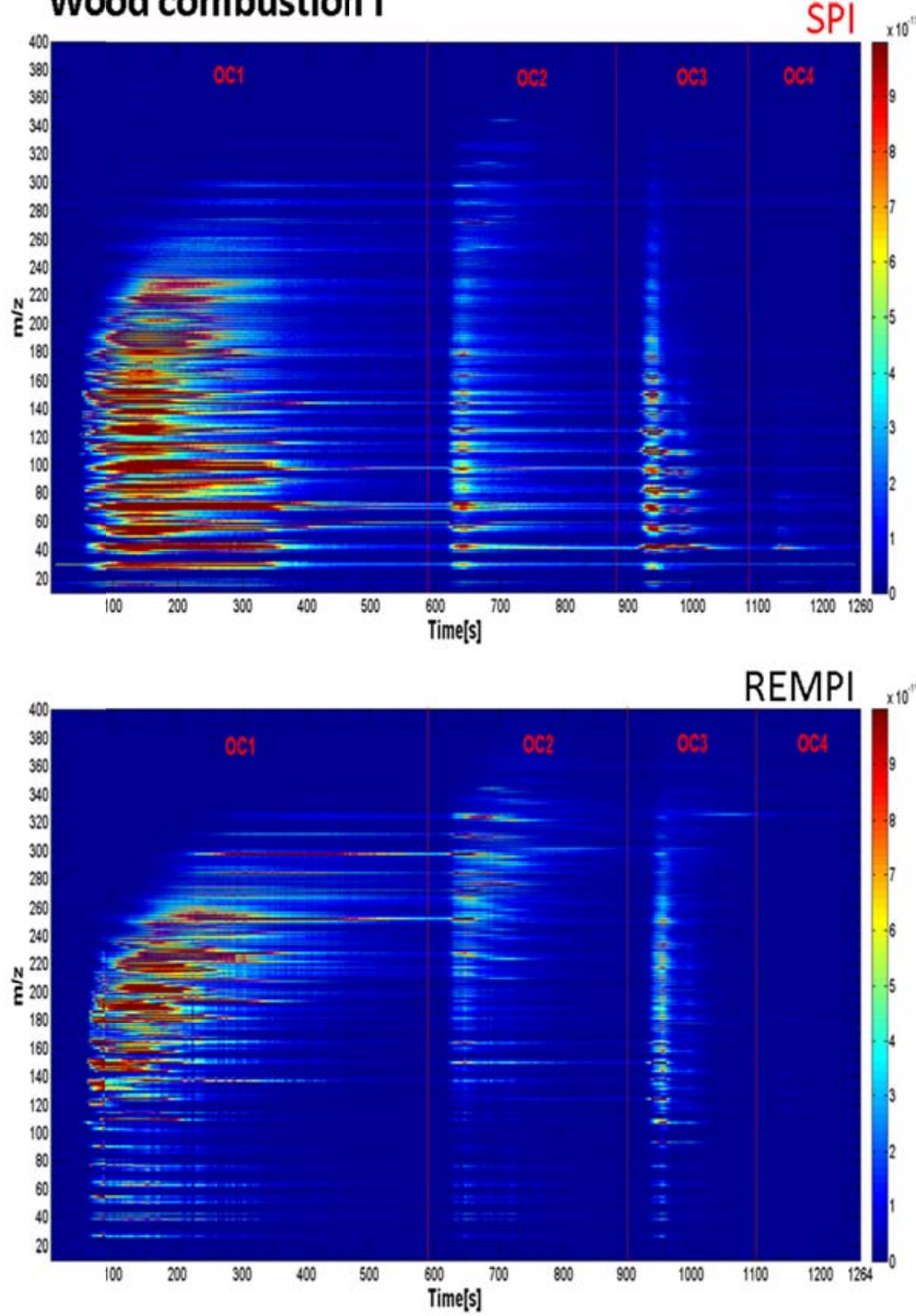

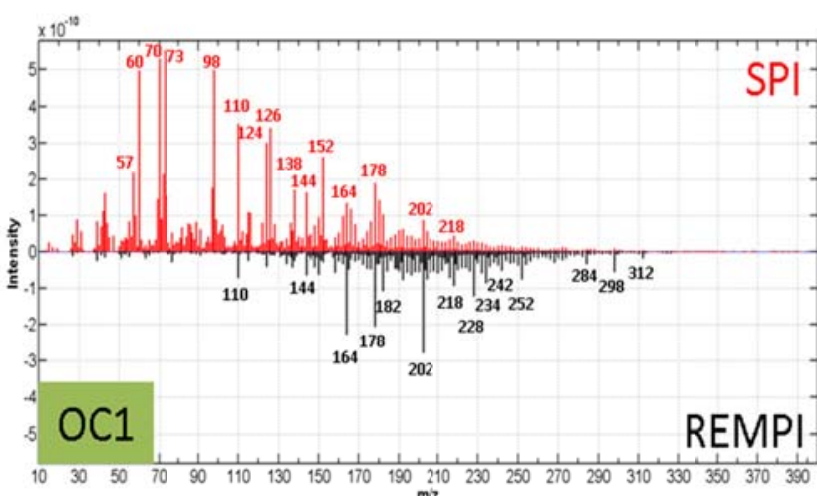$$
1010
$$
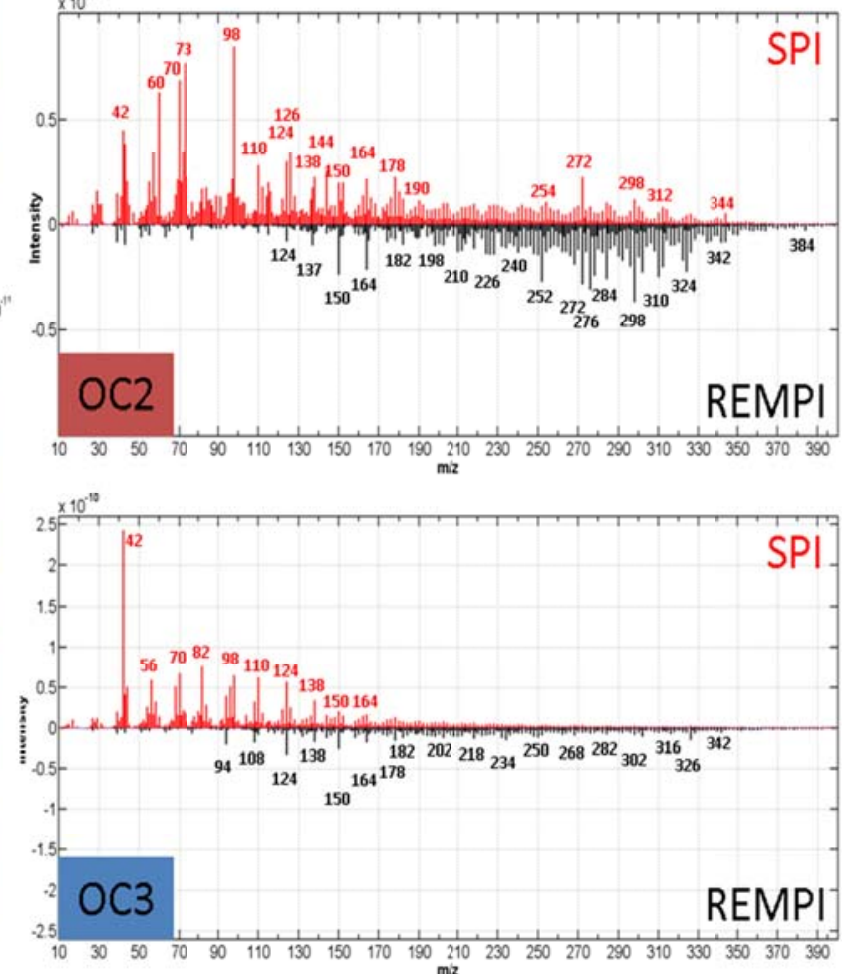
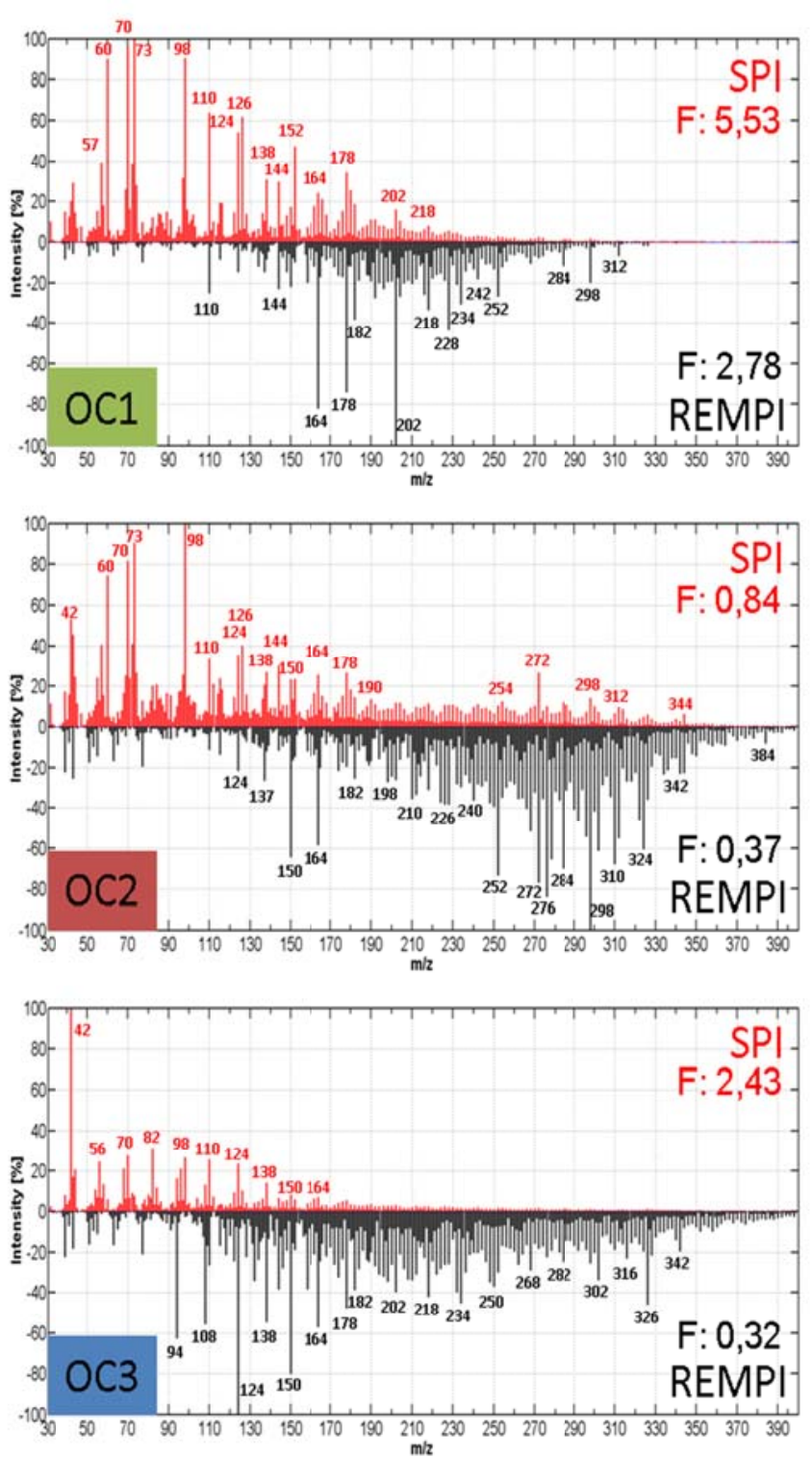

Figure S5: Two-dimensional mass spectrum of the course of signals ( $\mathrm{m} / \mathrm{z}$ ) over the four OC-steps (left) for SPI (top) and REMPI (bottom), mass spectra for the first three OC-steps (SPI-red, REMPI-black) (middle), mass spectra for the first three OC-steps normalized to $100 \%$ (right) of the wood combustion emission sample from the starting phase. Measured with the IMPROVE protocol. 

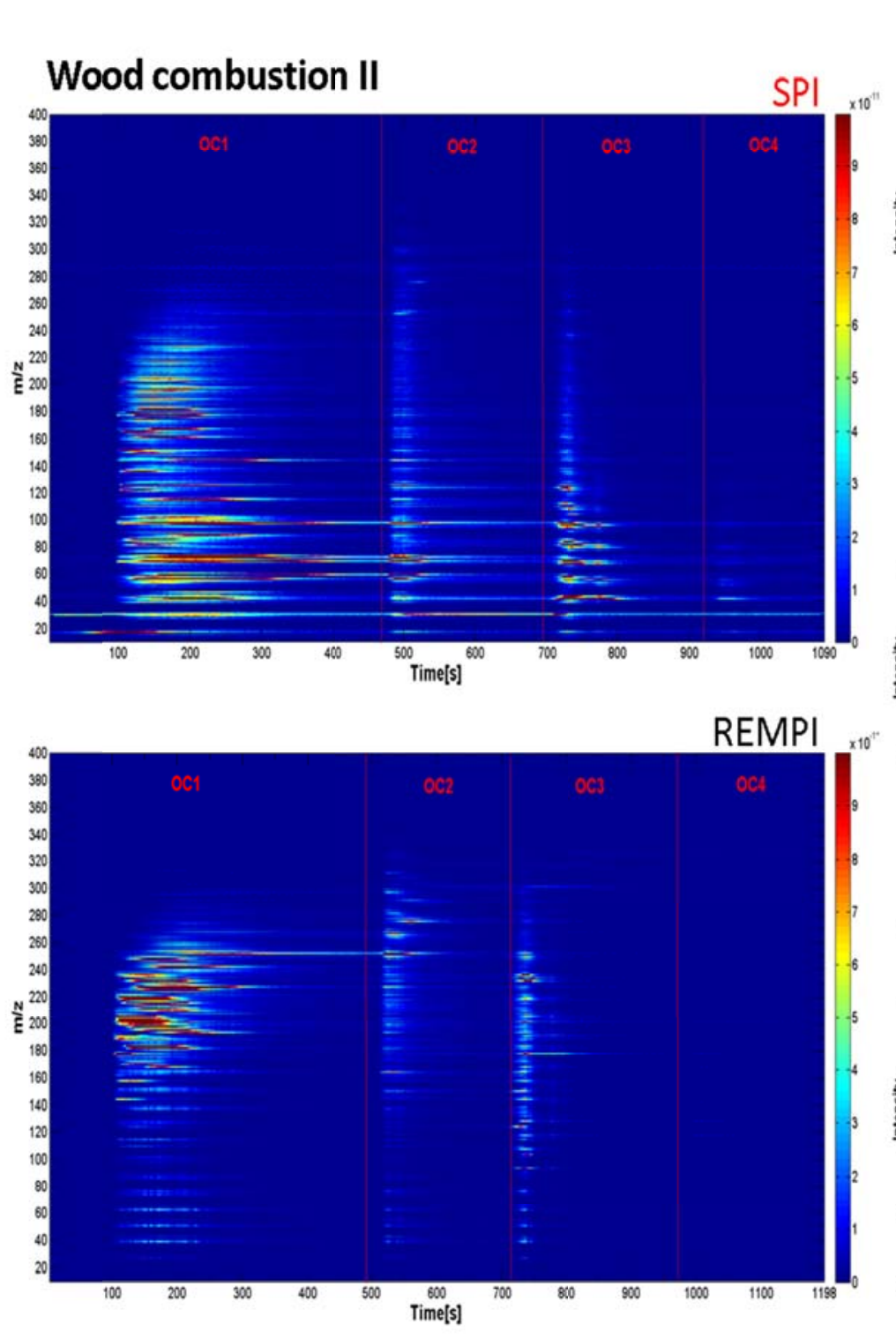
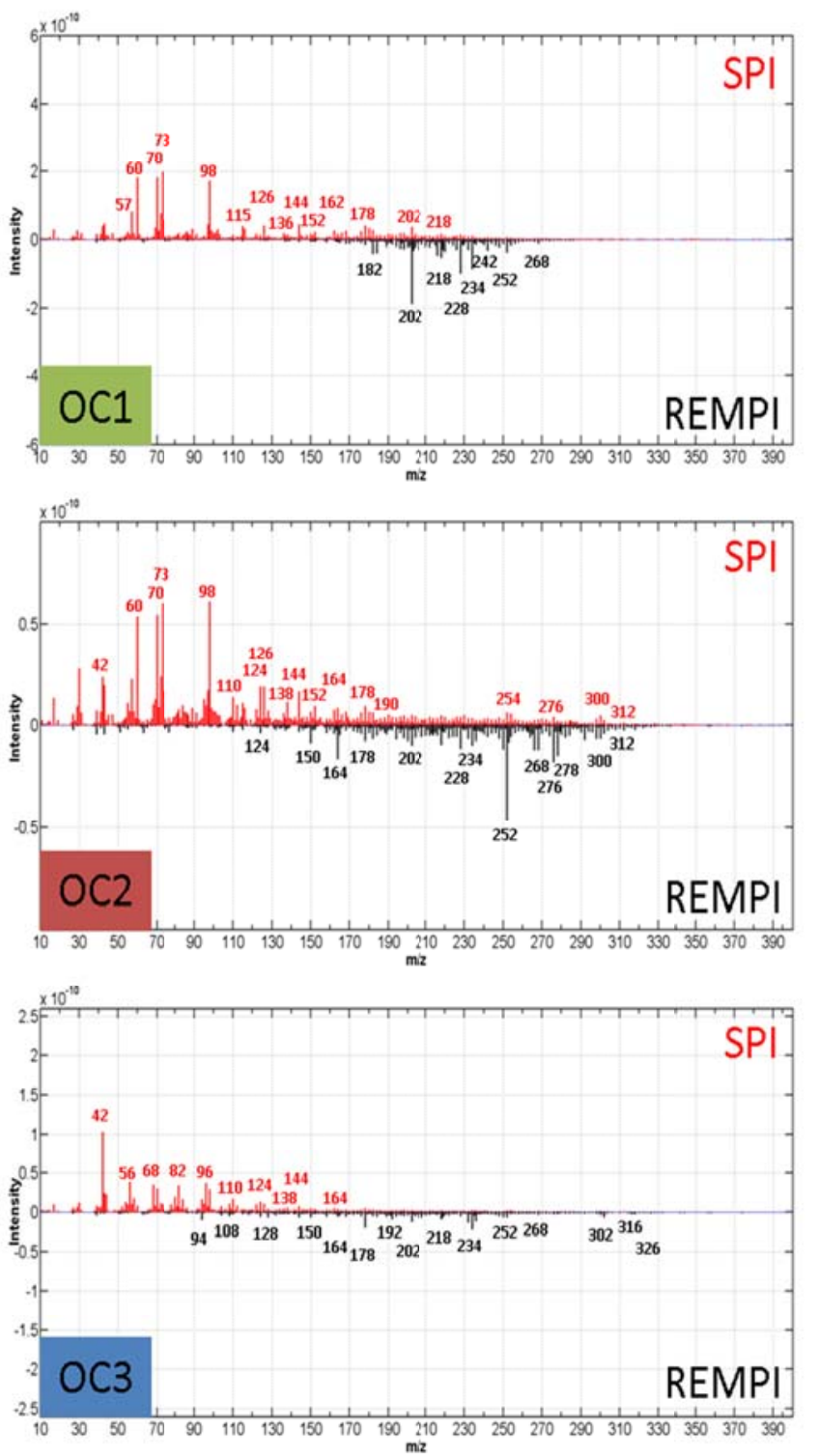
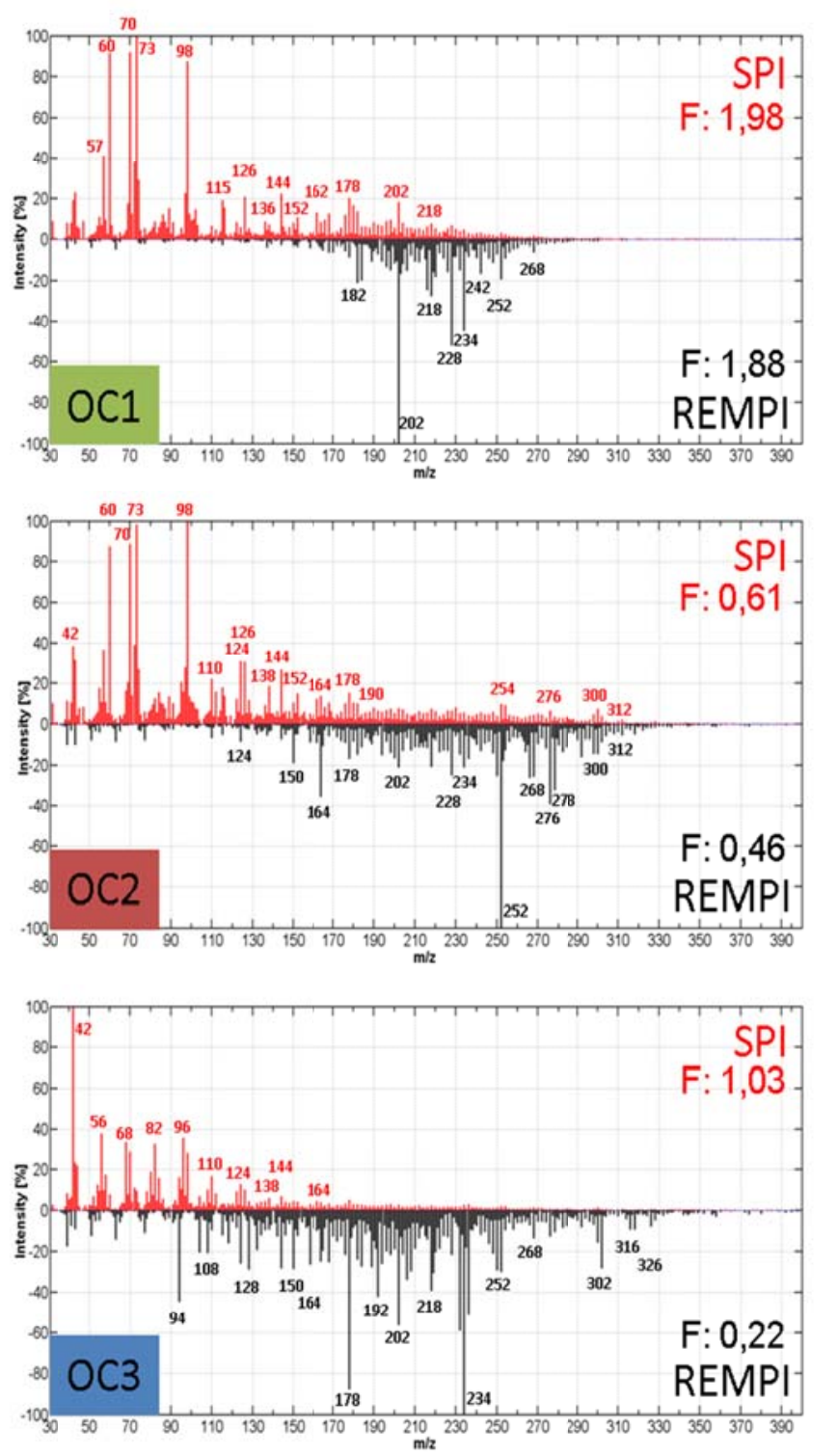

Figure S6: Two-dimensional mass spectrum of the course of signals ( $\mathrm{m} / \mathrm{z}$ ) over the four OC-steps (left) for SPI (top) and REMPI (bottom), mass spectra for the first three OC-steps (SPI-red, REMPI-black) (middle), mass spectra for the first three OC-steps normalized to $100 \%$ (right) of the wood combustion emission sample from the nominal load phase. Measured with the IMPROVE protocol. 


\section{Ispra ambient winter}
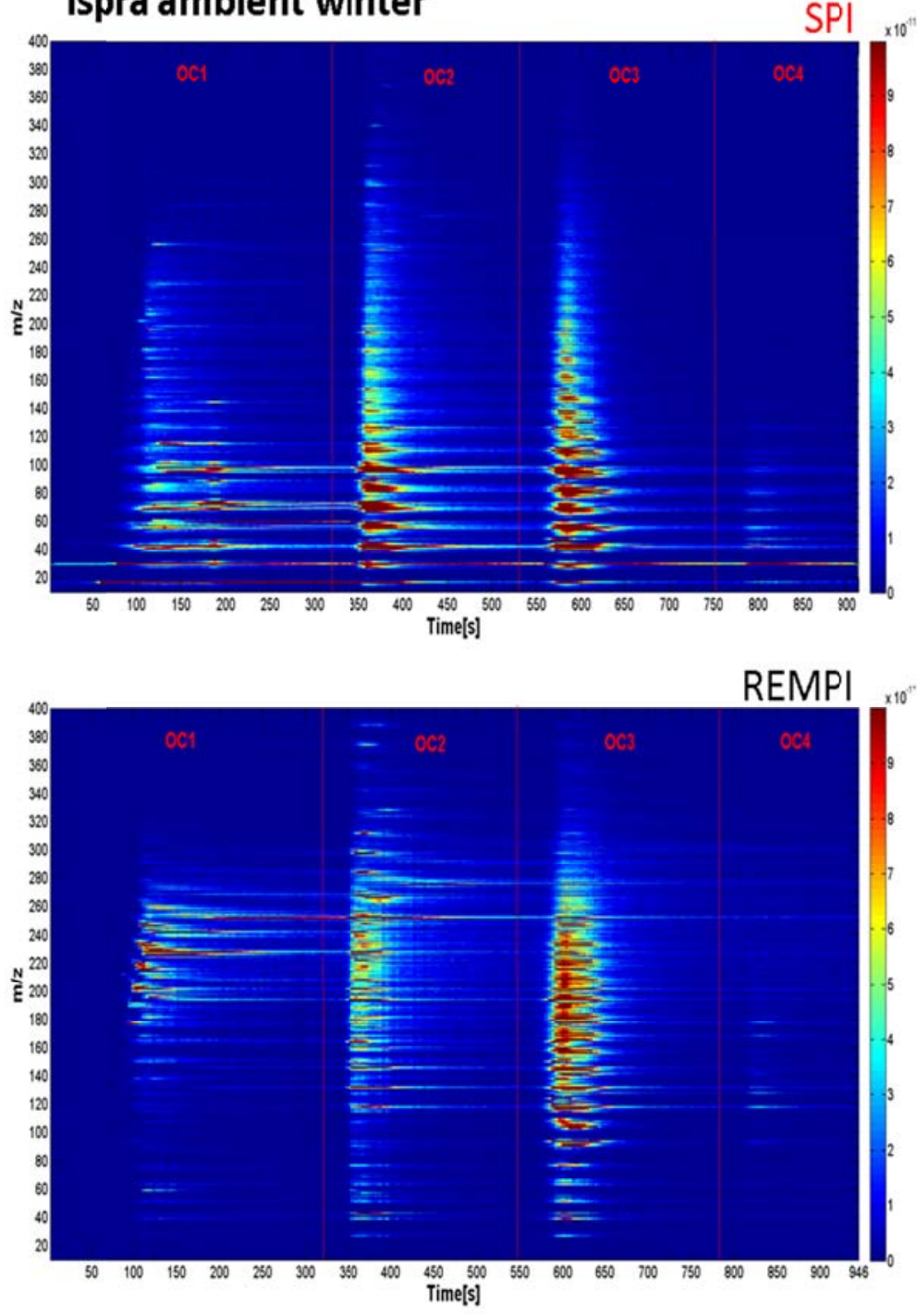
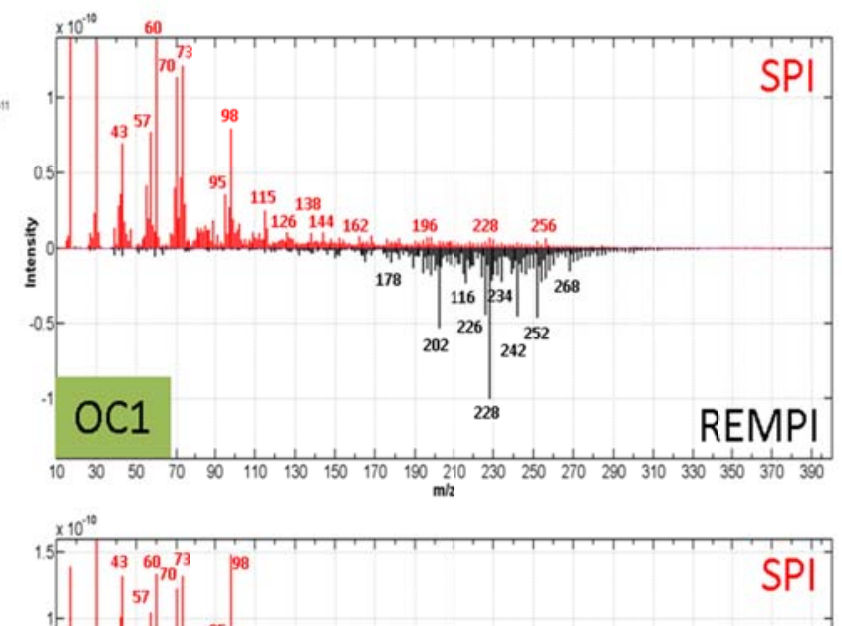

$0.5-1$ -
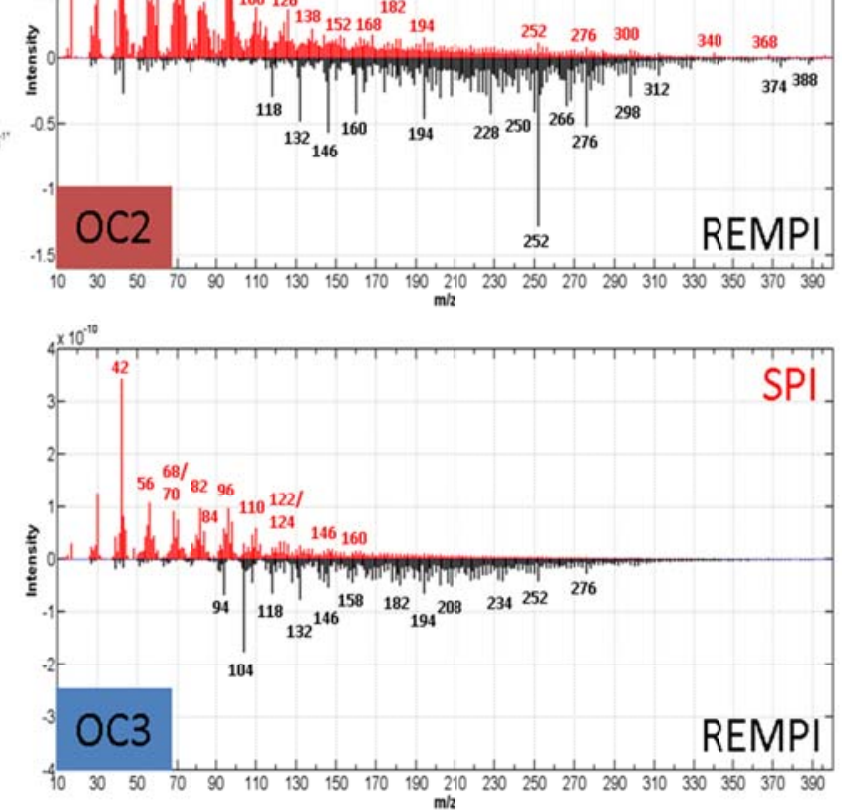
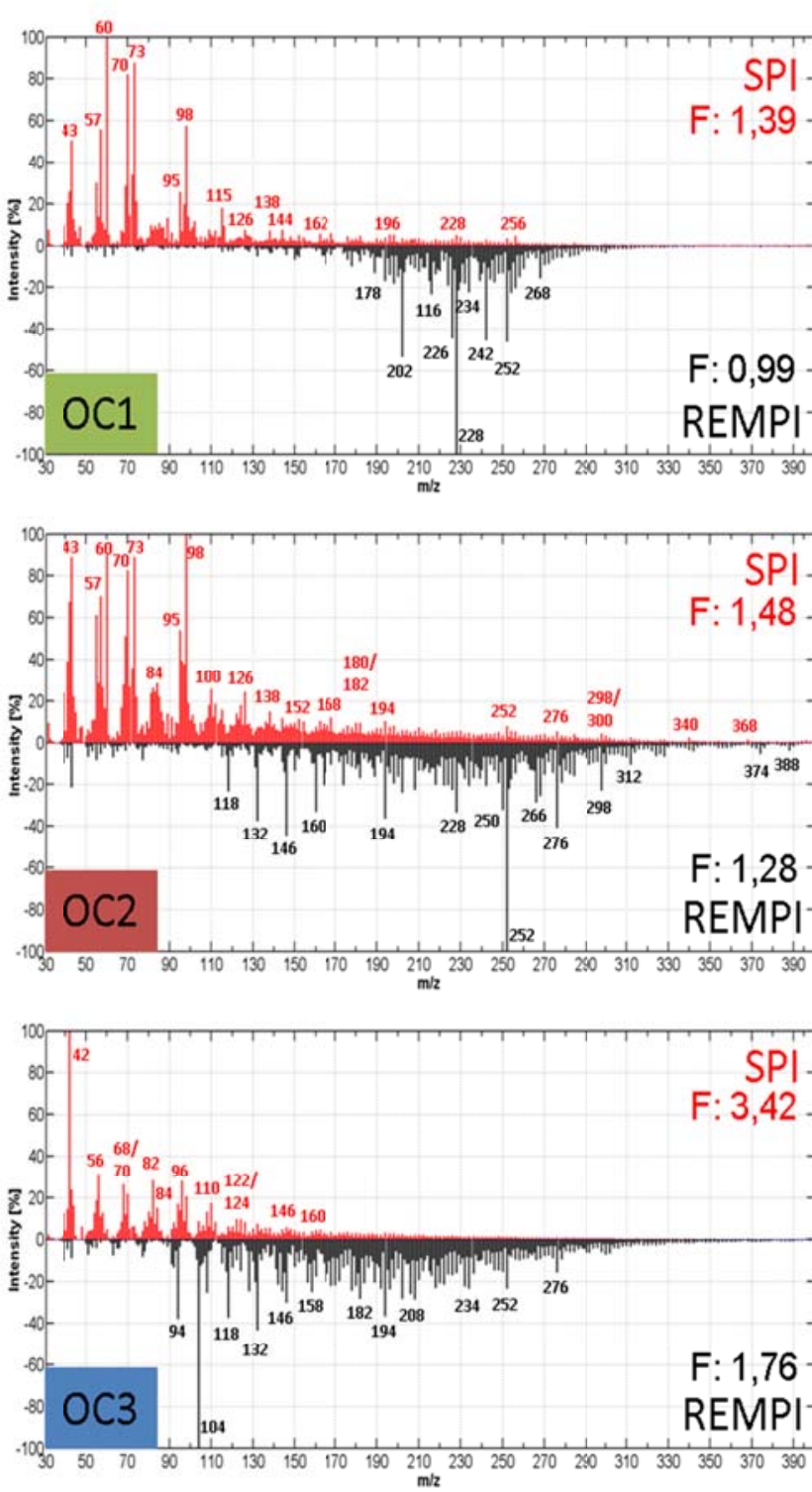

Figure S7: Two-dimensional mass spectrum of the course of signals (m/z) over the four OC-steps (left) for SPI (top) and REMPI (bottom), mass spectra for the first three OC-steps (SPI-red, REMPI-black) (middle), mass spectra for the first three OC-steps normalized to $100 \%$ (right) of the ambient winter sample from the rural background site in Ispra, Italy. Measured with the IMPROVE-A protocol. 

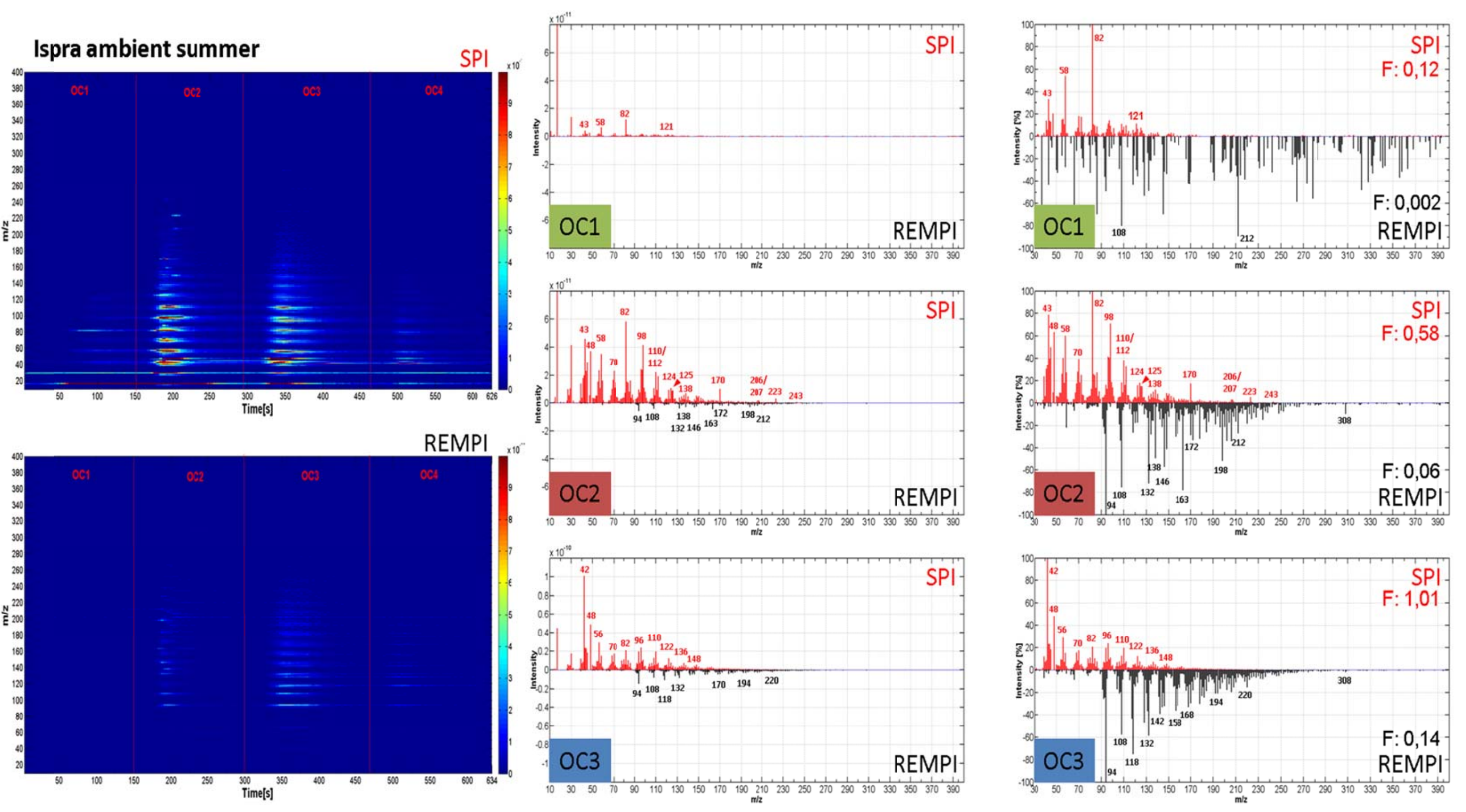

Figure S8: Two-dimensional mass spectrum of the course of signals ( $\mathrm{m} / \mathrm{z}$ ) over the four OC-steps (left) for SPI (top) and REMPI (bottom), mass spectra for the first three OC-steps (SPI-red, REMPI-black) (middle), mass spectra for the first three OC-steps normalized to $100 \%$ (right) of the ambient summer sample from the rural background site in Ispra, Italy. Measured with the IMPROVE-A protocol. 

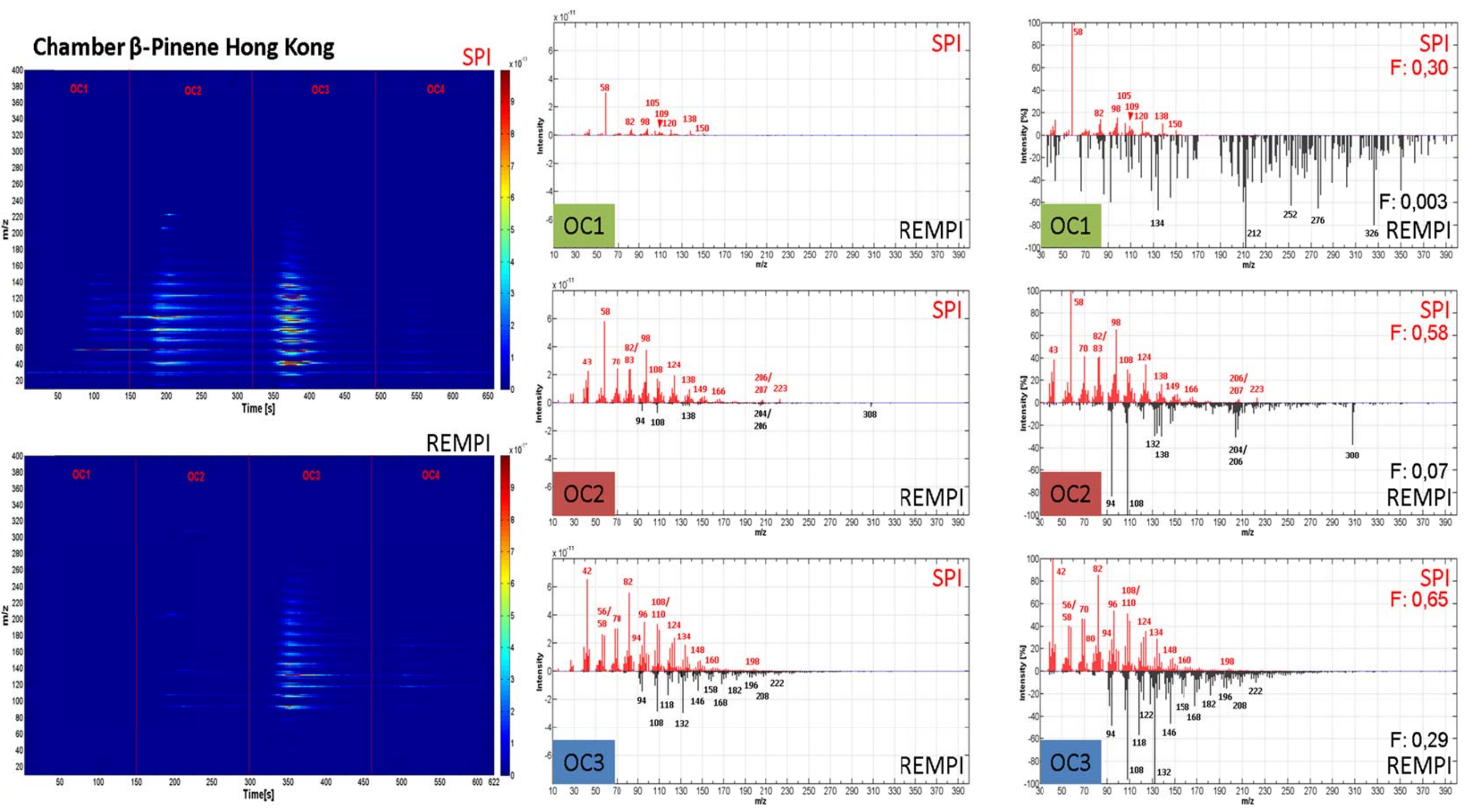

Figure S9: Two-dimensional mass spectrum of the course of signals $(\mathrm{m} / \mathrm{z})$ over the four OC-steps (left) for SPI (top) and REMPI (bottom), mass spectra for the first three OC-steps (SPI-red, REMPI-black) (middle), mass spectra for the first three OC-steps normalized to $100 \%$ (right) of the chamber experiment performed in Hong Kong with $\beta$-Pinene as a precursor. Measured with the IMPROVE-A protocol. 

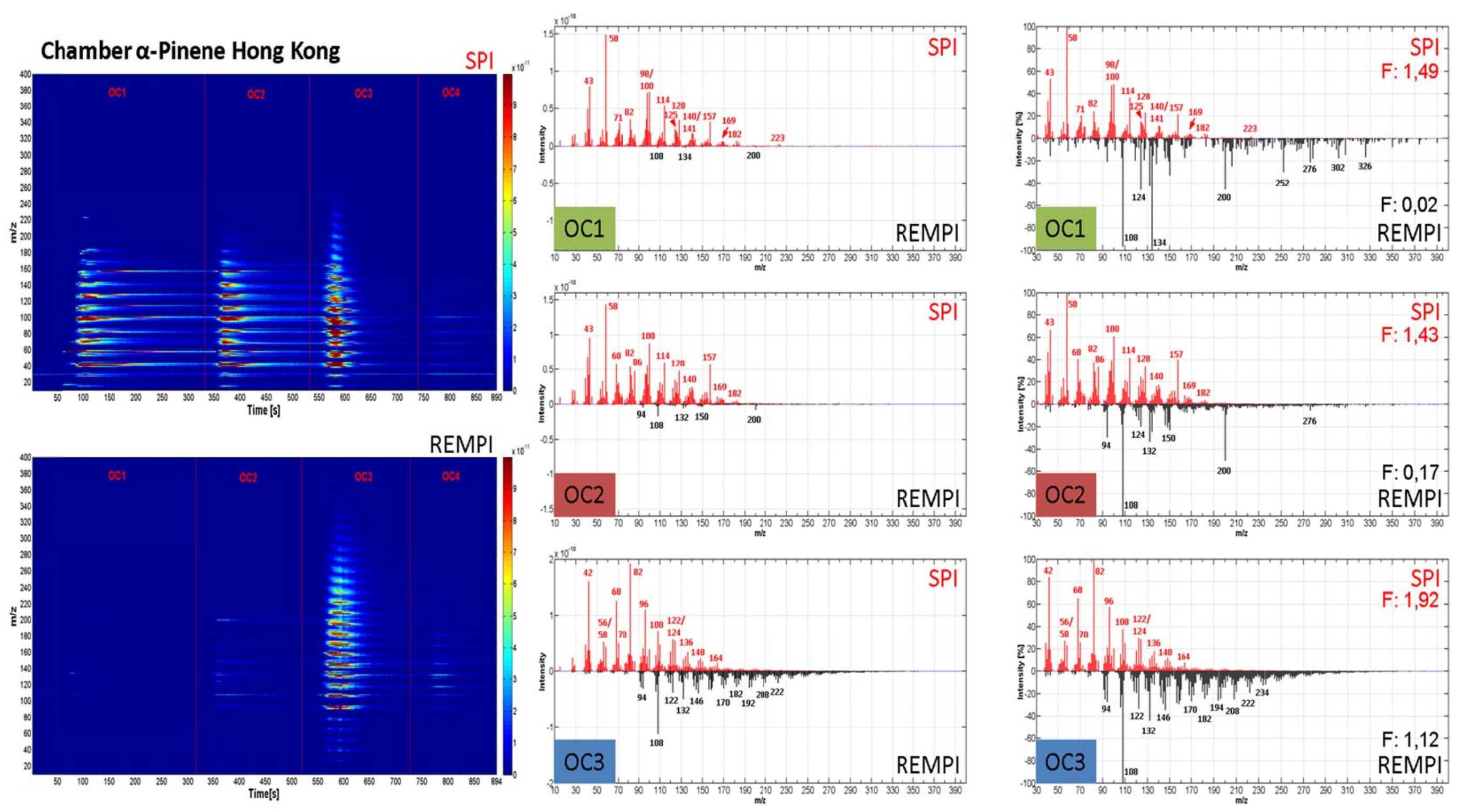

Figure S10: Two-dimensional mass spectrum of the course of signals ( $\mathrm{m} / \mathrm{z}$ ) over the four OC-steps (left) for SPI (top) and REMPI (bottom), mass spectra for the first three OC-steps (SPI-red, REMPI-black) (middle), mass spectra for the first three OC-steps normalized to $100 \%$ (right) of the chamber experiment performed in Hong Kong with $\alpha$-Pinene as a precursor. Measured with the IMPROVE-A protocol. 

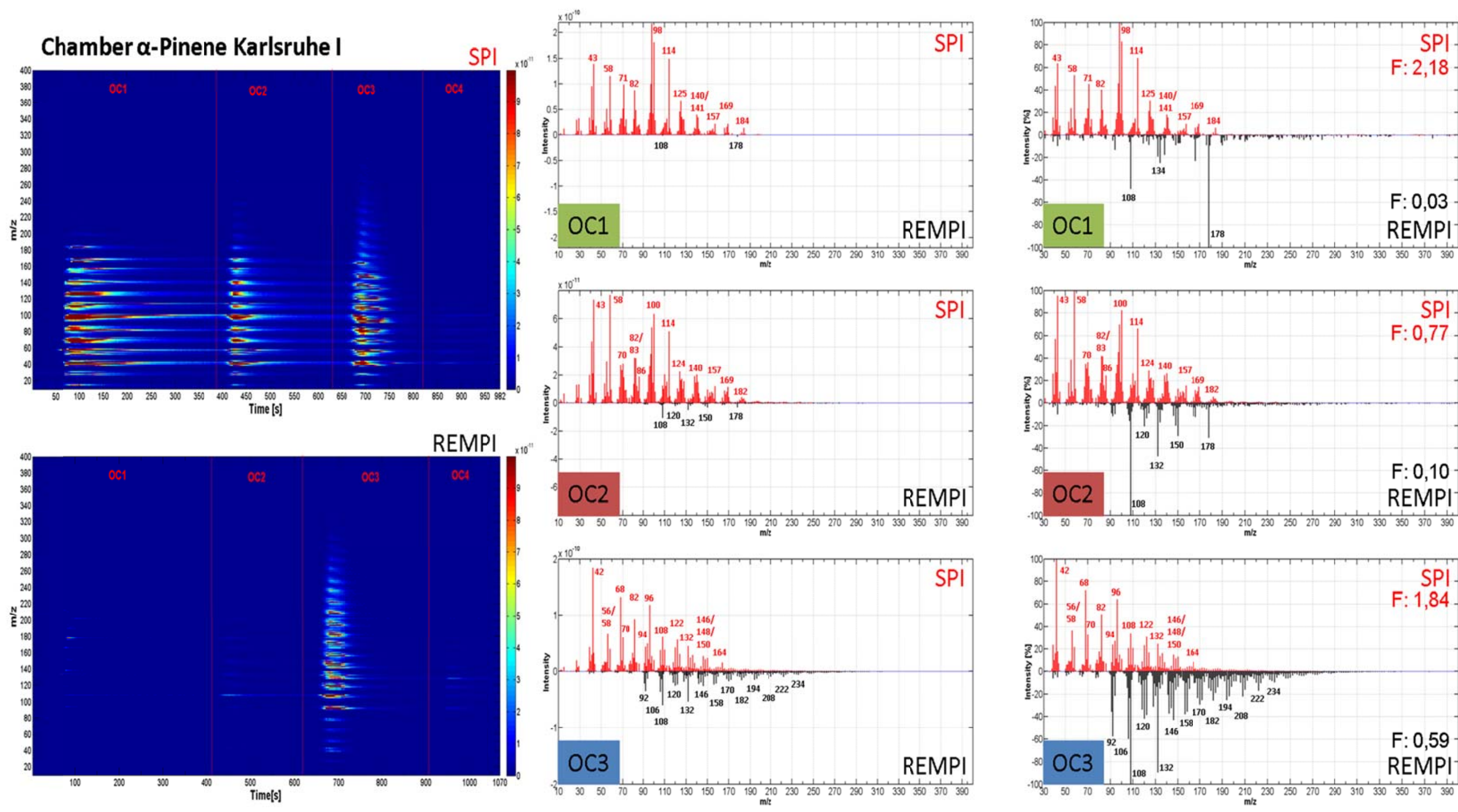

Figure S11: Two-dimensional mass spectrum of the course of signals ( $\mathrm{m} / \mathrm{z}$ ) over the four OC-steps (left) for SPI (top) and REMPI (bottom), mass spectra for the first three OC-steps (SPI-red, REMPI-black) (middle), mass spectra for the first three OC-steps normalized to $100 \%$ (right) of the chamber experiment performed in Karlsruhe with $\alpha$-Pinene as a precursor. Measured with the IMPROVE-A protocol. 

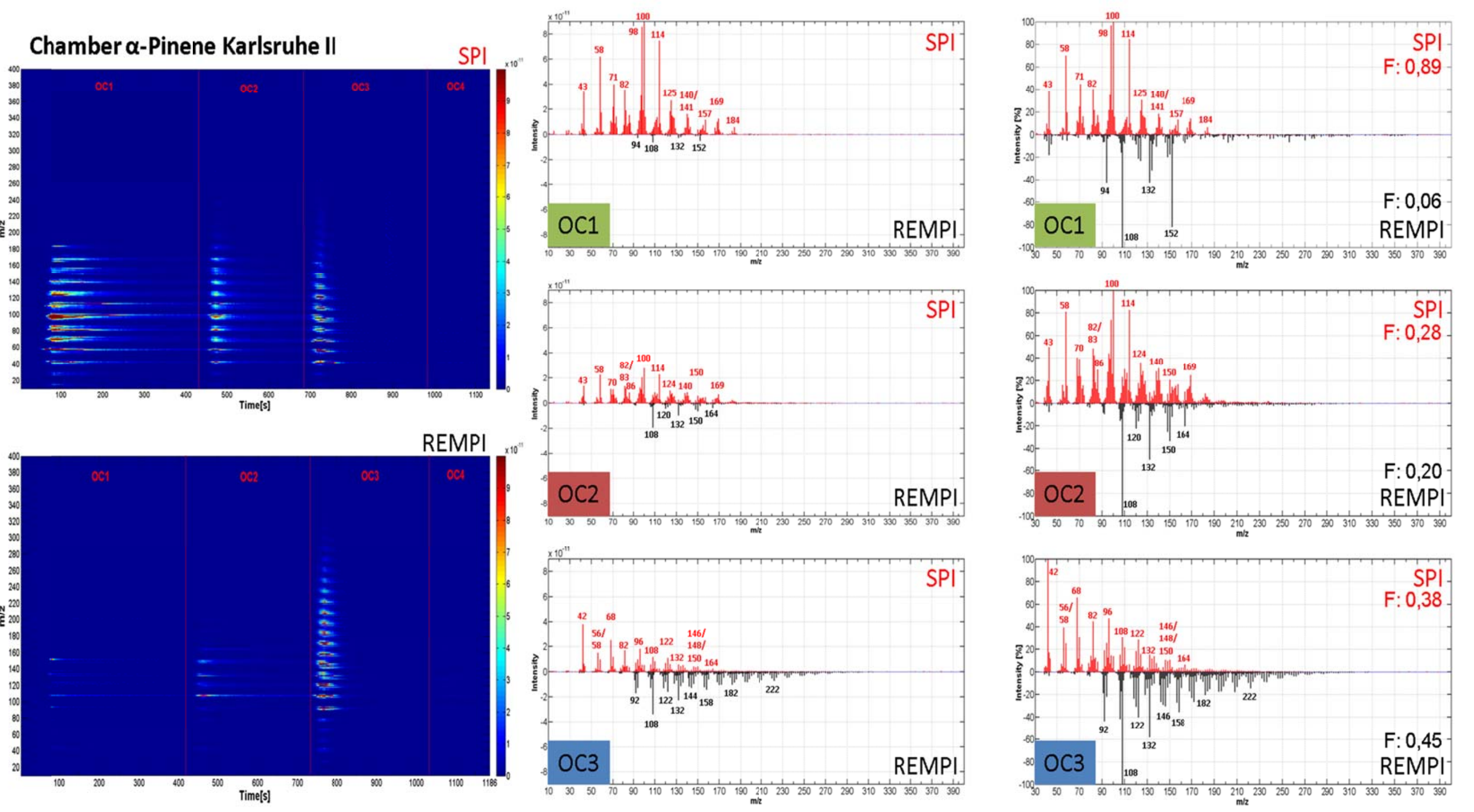

Figure S12: Two-dimensional mass spectrum of the course of signals ( $\mathrm{m} / \mathrm{z}$ ) over the four OC-steps (left) for SPI (top) and REMPI (bottom), mass spectra for the first three OC-steps (SPI-red, REMPI-black) (middle), mass spectra for the first three OC-steps normalized to $100 \%$ (right) of the chamber experiment performed in Karlsruhe with $\alpha$-Pinene as a precursor. Measured with the IMPROVE-A protocol. 

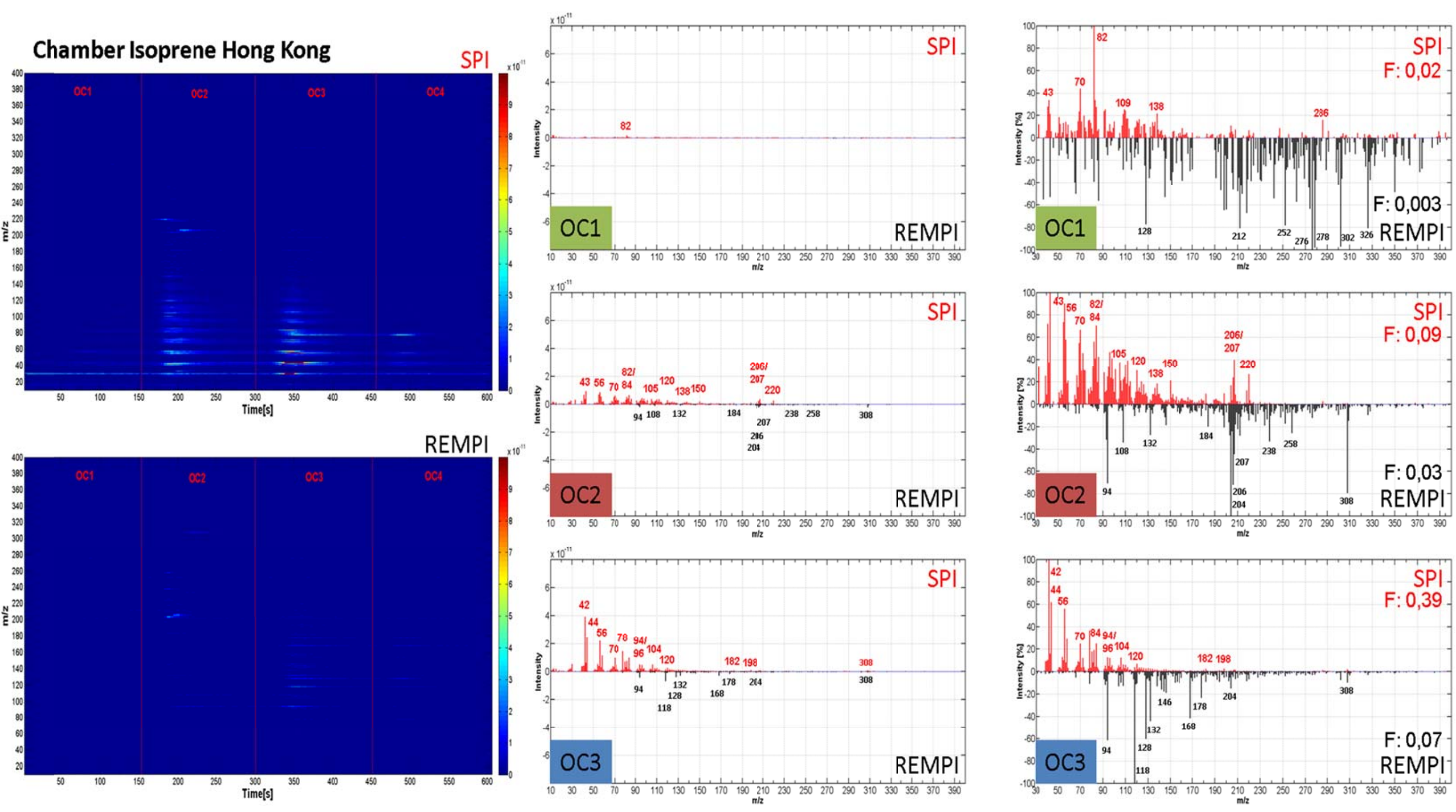

Figure S13: Two-dimensional mass spectrum of the course of signals ( $\mathrm{m} / \mathrm{z}$ ) over the four OC-steps (left) for SPI (top) and REMPI (bottom), mass spectra for the first three OC-steps (SPI-red, REMPI-black) (middle), mass spectra for the first three OC-steps normalized to $100 \%$ (right) of the chamber experiment performed in Hong Kong with Isoprene as a precursor. Measured with the IMPROVE-A protocol. 

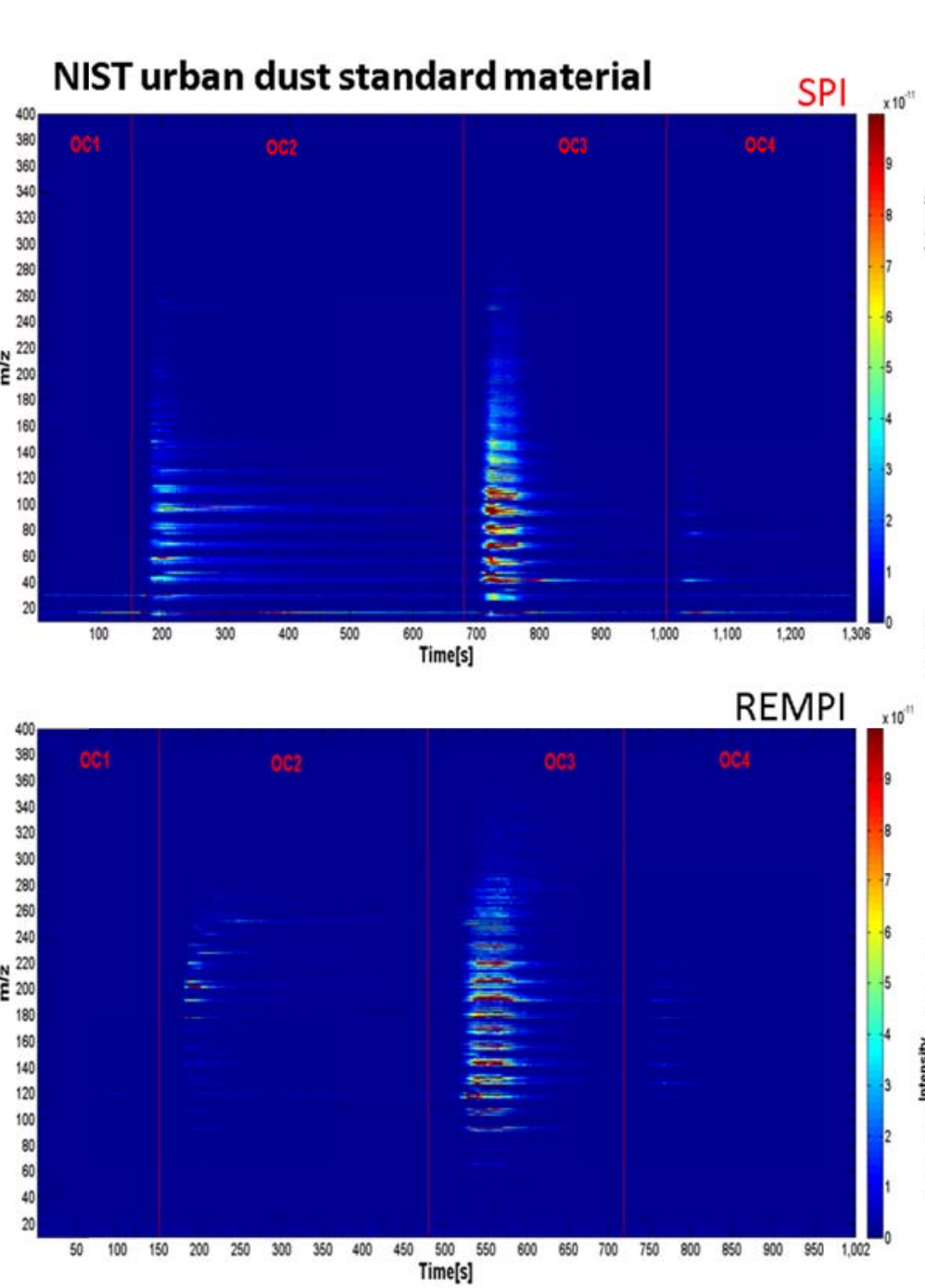
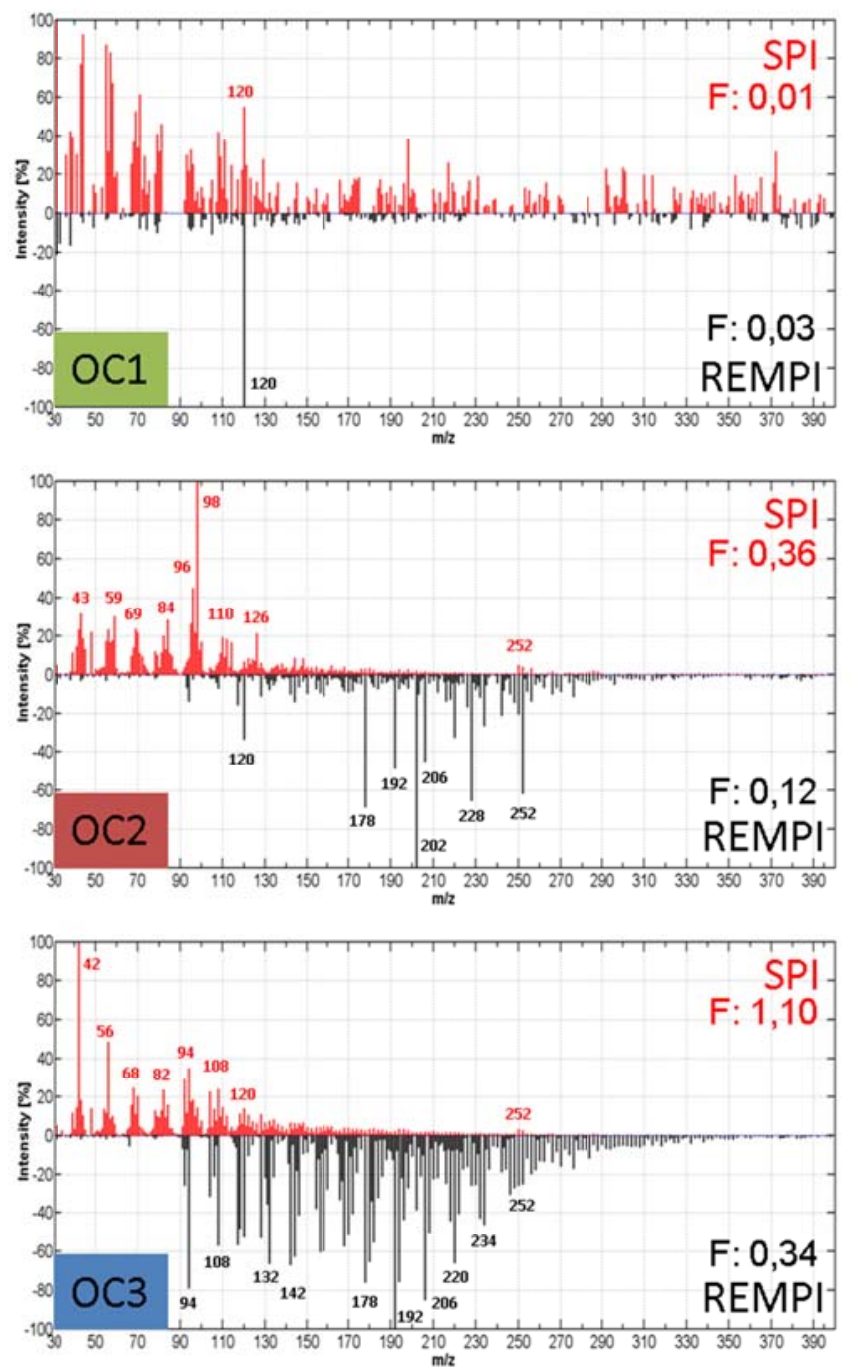

Figure S14: Two-dimensional mass spectrum of the course of signals ( $\mathrm{m} / \mathrm{z}$ ) over the four OC-steps (left) for SPI (top) and REMPI (bottom), mass spectra for the first three OC-steps (SPI-red, REMPI-black) (middle), mass spectra for the first three OC-steps normalized to $100 \%$ (right) of the NIST urban dust standard material. Measured with the IMPROVE-A protocol. 
Table S3: proposals of the most likely compounds for the signals found in the samples analyzed, whereby (?) means that no suitable compound was found in the literature, $(-)$ mean: not present in the sample, $(+)$ present, $(++)$ dominant signal.

\begin{tabular}{|c|c|c|c|c|c|c|c|c|c|c|c|c|c|c|c|c|c|}
\hline \multirow[t]{2}{*}{$\mathrm{m} / \mathrm{z}$} & \multirow[t]{2}{*}{ structure proposals } & \multicolumn{2}{|c|}{$\begin{array}{l}\text { samples } \\
\text { gasoline }\end{array}$} & \multicolumn{2}{|c|}{ diesel } & \multicolumn{2}{|c|}{ wood combustion } & \multicolumn{2}{|c|}{ Italy winter } & \multicolumn{2}{|c|}{ Italy summer } & \multicolumn{2}{|c|}{$\beta$-Pinene HK } & \multicolumn{2}{|c|}{$\alpha$-Pinene HK } & \multicolumn{2}{|c|}{$\alpha$-Pinene KR } \\
\hline & & SPI & REMPI & SPI & REMPI & SPI & REMPI & SPI & REMPI & SPI & REMPI & SPI & REMPI & SPI & REMPI & SPI & REMPI \\
\hline 42 & propene & ++ & - & ++ & - & ++ & - & ++ & - & ++ & - & ++ & - & ++ & - & ++ & - \\
\hline 43 & alkyl fragment & + & - & + & - & - & - & ++ & - & ++ & - & + & - & ++ & - & ++ & - \\
\hline 44 & acetaldehyde & ++ & - & - & - & - & - & - & - & - & - & - & - & - & - & - & - \\
\hline 48 & methylsulfide & - & - & - & - & - & - & - & - & ++ & - & - & - & - & - & - & - \\
\hline 55 & alkene fragment & + & - & + & - & - & - & ++ & - & - & - & - & - & - & - & - & - \\
\hline 56 & butene, propenal, acrolein & + & - & ++ & - & ++ & - & ++ & - & + & - & ++ & - & + & - & + & - \\
\hline 57 & alkyl fragment & - & - & + & - & + & - & ++ & - & - & - & - & - & - & - & - & - \\
\hline 58 & acetone, propanal, glyoxal, ... & + & - & - & - & - & - & - & - & ++ & - & ++ & - & ++ & - & ++ & - \\
\hline 60 & wood combustion fragment $(\mathrm{C} 2 \mathrm{H} 4 \mathrm{O} 2+)$, glycolaldehyde & - & - & - & - & ++ & - & ++ & - & - & - & - & - & - & - & - & - \\
\hline 70 & pentene, methylbutene, methacrolein, methyl vynil ketone, ... & + & - & + & - & ++ & - & ++ & - & + & - & ++ & - & + & - & + & - \\
\hline 73 & wood combustion fragment $(\mathrm{C} 3 \mathrm{H} 5 \mathrm{O} 2+)$, butylamine & - & - & - & - & ++ & - & ++ & - & - & - & - & - & - & - & - & - \\
\hline 78 & benzene, ? & + & - & + & - & - & - & - & - & - & - & - & - & - & - & - & - \\
\hline 82 & Methylfuran, methylpyrazole, ... & - & - & + & - & ++ & - & ++ & - & ++ & - & ++ & - & ++ & - & ++ & - \\
\hline 83 & $?$ & - & - & - & - & - & - & + & - & - & - & + & - & + & - & + & - \\
\hline 84 & cyclohexane, methylbutenal, hexene,$\ldots$ & - & - & + & - & - & - & + & - & + & - & - & - & - & - & - & - \\
\hline 86 & hexane, methylbutanal, ... & + & - & - & - & - & - & - & - & + & - & - & - & + & - & + & - \\
\hline 92 & toluene & - & - & - & - & - & - & - & - & - & - & - & - & - & + & - & + \\
\hline 94 & phenol,vinylfuran, methylpyrazine, ... & + & ++ & + & ++ & + & + & + & ++ & + & + & + & + & + & + & - & + \\
\hline 95 & dimethylpyrrole, formylpyrrole, ... & - & - & - & - & - & - & ++ & - & - & - & - & - & + & - & - & - \\
\hline 96 & diemthylfuran, furfural, dimethylpyrazole, ... & - & - & + & - & + & - & ++ & - & + & - & ++ & - & ++ & - & ++ & - \\
\hline
\end{tabular}




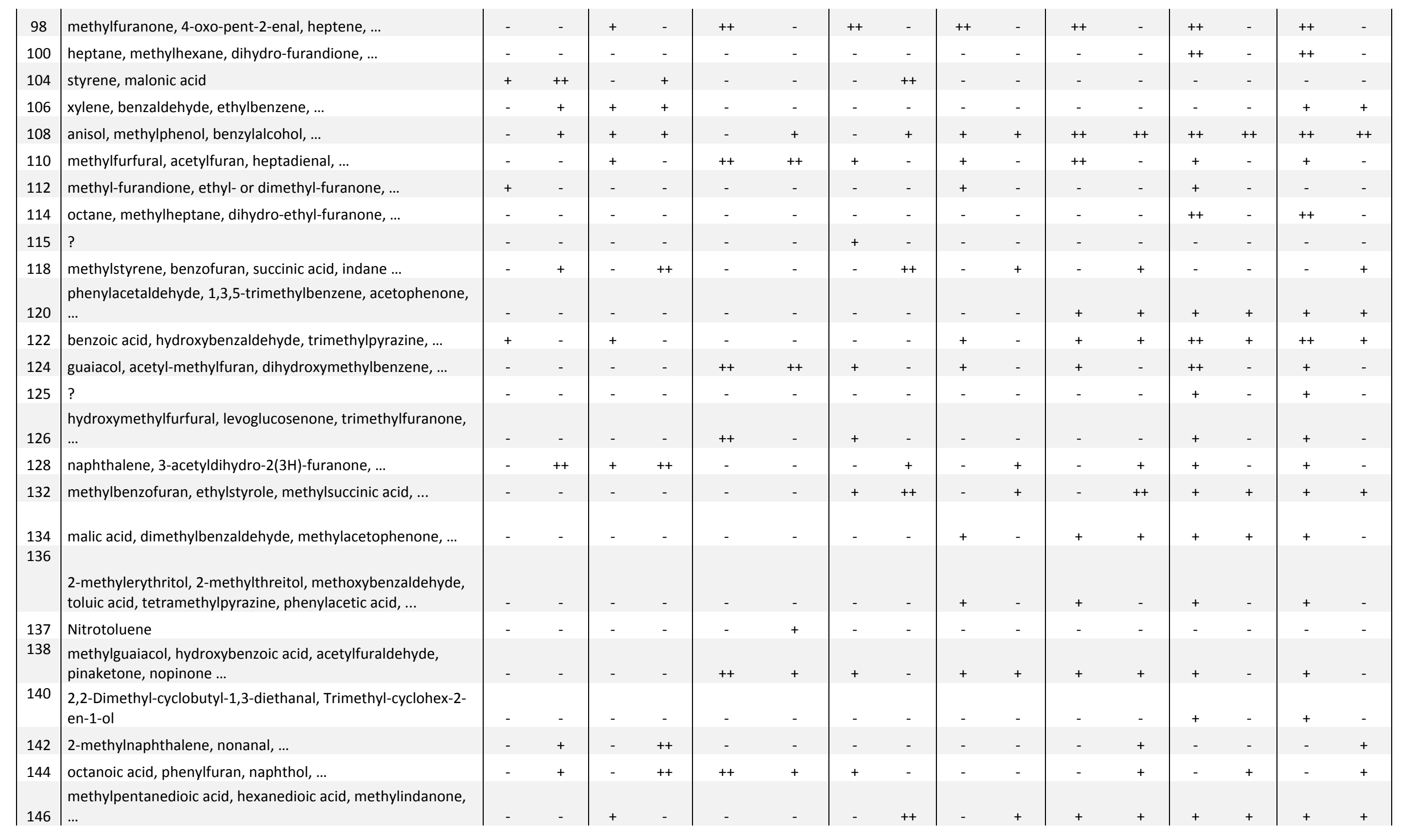




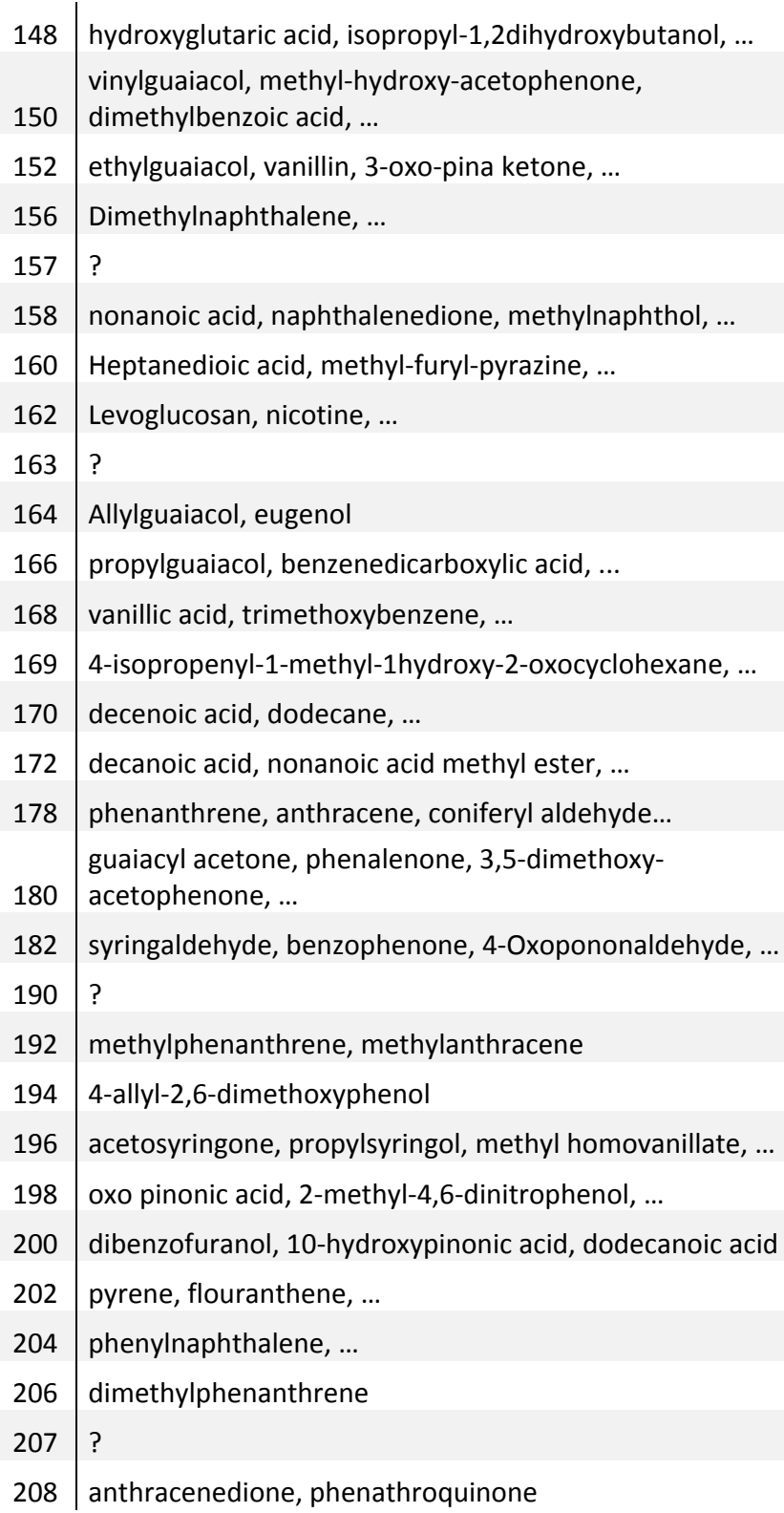




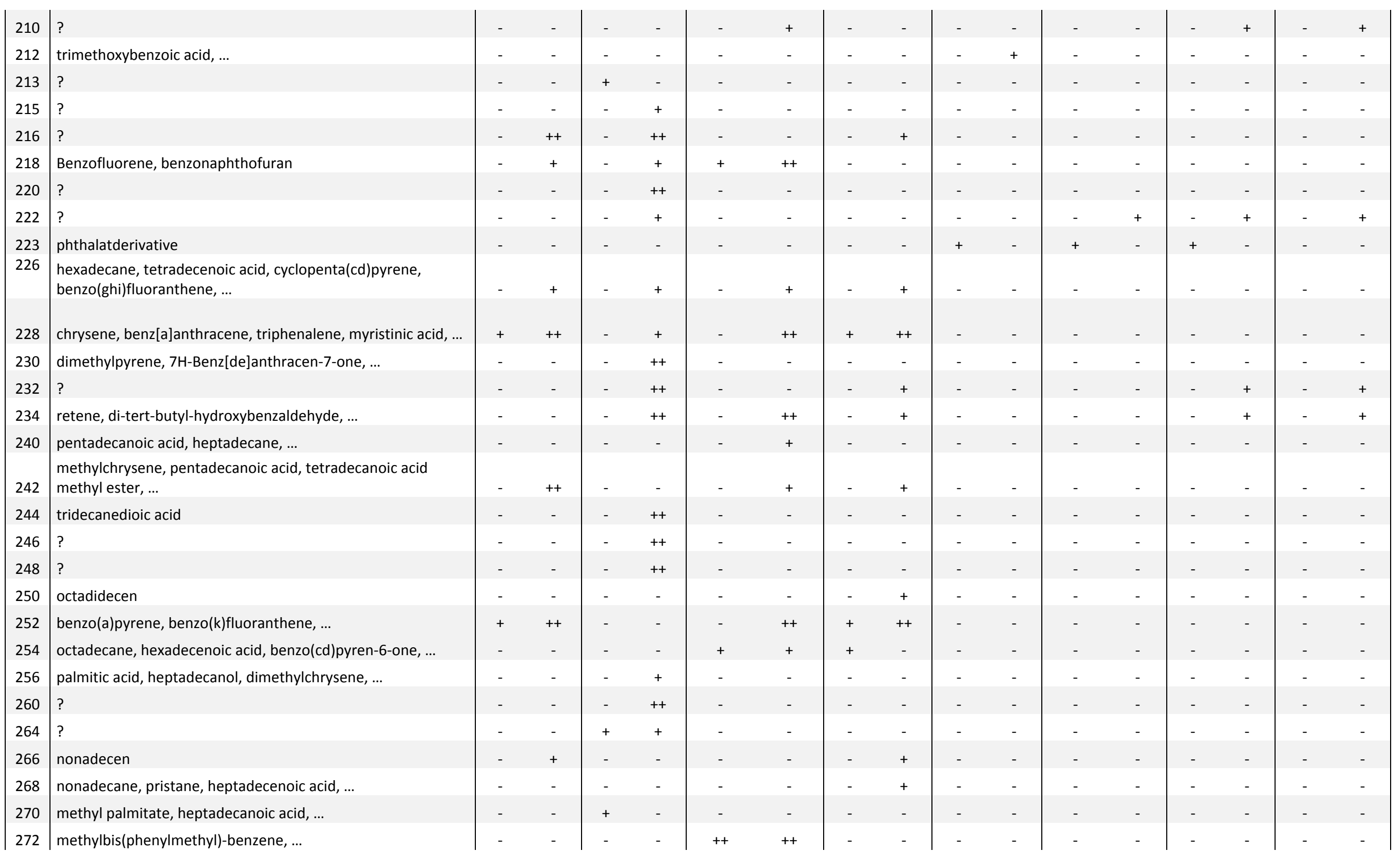




\begin{tabular}{|c|c|c|c|c|c|c|c|c|c|c|c|c|c|c|c|c|c|}
\hline 276 & benzo(ghi)perylene, indeno(1,2,3-cd)pyrene, ... & + & ++ & - & + & - & ++ & + & + & - & - & - & - & - & - & - & - \\
\hline 278 & dibenz[a,h]anthracene & - & + & - & - & - & + & - & - & - & - & - & - & - & - & - & - \\
\hline 284 & heptadecanoic acid methyl ester, stearic acid, ... & - & - & - & - & - & ++ & - & - & - & - & - & - & - & - & - & - \\
\hline 296 & heneicosane & - & - & + & - & - & - & - & - & - & - & - & - & - & - & - & - \\
\hline 298 & abietatetraenoic acid, octadecanoic acid methyl ester, ... & - & - & - & - & + & ++ & - & + & - & - & - & - & - & - & - & - \\
\hline 300 & coronene, dehydroabietic acid & - & ++ & - & - & + & + & + & + & - & - & - & - & - & - & - & - \\
\hline 302 & pimaric acid, abietic acid & - & ++ & - & - & + & - & + & - & - & - & - & - & - & - & - & - \\
\hline 310 & ethyl oleate, docosane, ... & - & - & - & - & - & + & - & - & - & - & - & - & - & - & - & - \\
\hline 312 & methylabietatetraenoate, eicoscanoic acid, ethyl stearate, ... & - & - & - & - & + & ++ & + & + & - & - & - & - & - & - & - & - \\
\hline 322 & $?$ & - & - & - & - & - & + & - & - & - & - & - & - & - & - & - & - \\
\hline 324 & Tricosane, docosanal, ... & - & - & - & - & - & + & - & - & - & - & - & - & - & - & - & - \\
\hline 326 & $?$ & - & - & - & - & - & + & - & - & - & - & - & - & - & - & - & - \\
\hline 340 & docosanoic acid, tricosanol, ... & - & - & - & - & + & - & + & - & - & - & - & - & - & - & - & - \\
\hline 344 & deoxomatairesinol & - & - & - & - & + & + & - & - & - & - & - & - & - & - & - & - \\
\hline 368 & tetracosanoic acid & - & - & - & - & - & - & - & - & - & - & - & - & - & - & - & - \\
\hline 374 & $?$ & - & - & - & - & - & - & - & + & - & - & - & - & - & - & - & - \\
\hline 384 & deisopropyldehydroabietic acid & - & - & - & - & - & + & - & - & - & - & - & - & - & - & - & - \\
\hline 388 & $?$ & - & - & - & - & - & - & - & + & - & - & - & - & - & - & - & - \\
\hline
\end{tabular}

